\title{
GRAVELLING TEST FOR RAIL WINDSHIELD
}

\author{
Adam Bilar, Pawel Boguszewicz, Witold Perkowski \\ Centre of Space Technologies, Institute of Aviation \\ Krakowska Av. 110/114, 02-256 Warsaw, Poland \\ tel.: + 48228460011 ex. 465, ex. 345, ex. 373, fax: +48228464432 \\ e-mail:adam.bilar@ilot.edu.pl,pawel.boguszewicz@ilot.edu.pl \\ witold.perkowski@ilot.edu.pl
}

\begin{abstract}
During preliminary tests of locomotive windshield resistance to gravelling, according to the applicable standard $P N-E N$ 15152:2007, carried out in the Institute of Aviation, the problem of the projectile overturning after leaving the cannon barrel was detected. Three tests recorded with a high-speed camera are presented in this article. This problem was found to be due to the incorrect projectile geometry.

In order to stabilise the trajectory of the projectile, four types of projectiles were made using military experience. All of them meet relevant test conditions from the point of view of the test piece (locomotive windshield). The best projectile modification was obtained in the tests with an average deviation of the expected impact angle of $11^{\circ}$ compared to $75^{\circ}$ for a projectile made exactly according to the guidelines of the standard. Each of 22 modified projectiles hit the test piece with the tip, while out of the 12 original projectiles only 4 hit the test piece with the tip. The impact test results confirm that it is possible to perform a gravelling test according to the standard concept, but it seems necessary to clarify the standard requirements and to modify the projectile shape.

The article contains a proposal for the projectile modification and highlights inaccuracies in the standard concerning the gravelling test.
\end{abstract}

Keywords: Impact test, locomotive windshield, gravelling, projectile overturning, rail standard

\section{Introduction}

Modern rail vehicles move at ever-increasing speeds. Carriers rival each other in offering fast, comfortable and safe transport between agglomerations. According to international rules, the driver's safety is a fundamental issue. For example, the UIC 651 [8] leaflet, similarly to PN-EN 15152:2007 standard [6] requires a one-kilogram projectile test. Such an impact test has been carried out at the Institute of Aviation for years [7].

As the maximum train speeds increase, new safety hazards arise, which are minimised by appropriate regulations, standards, etc. One of these risks occurring in the rail transport is the impact of small particles (e.g. gravel) on the driver's windscreen cab. With current windshield technologies, it does not pose a life or health threat neither the train driver nor the people in the cab; however, the standard PN-EN 15152:2007 [6] specifies the requirements for testing the windscreen resistance to the above-mentioned occurrence by means of the so-called gravelling test. Due to different conditions of rail vehicles use (infrastructure, speed, aerodynamics of the vehicle's head), the standard [6] in section 4.2.12 makes the gravelling test dependent on the user/buyer's decision.

In the course of preparation for a gravelling test at the Institute of Aviation, it was discovered that the projectile presented in the standard [6] generally did not hit the glass with the intended apex. The analysis of the projectile geometry showed that the centre of mass lies behind its aerodynamic centre, which, with the slight disturbance, causes its rotational movement. Several modifications of the projectile were tested, which retained all relevant parameters of the gravelling test included in the standard [6]. The purpose of the modified projectile tests was to verify the assumption that moving the gravity centre in front of the centre of pressure and maintaining their 
proper mutual distance will eliminate the phenomenon of the projectile overturning and will cause that its axis at the moment of impact will be close to perpendicularity to the tested sample surface.

The next paragraph contains the extract form the standard [6] describing gravelling test requirements.

\section{The requirements of the standard PN-EN 15152:2007 (sections 4.2.12 and 6.2.9)}

Source: Standard PN-EN 15152:2007. Sections:

\subsubsection{Resistance to repeated impact from small particles (gravelling)}

The risk of this type of damage will vary according to train speed, the aerodynamic conditions on the exterior of the driving cab and the constructions of the infrastructure on which the train will operate. It is therefore the choice of the customer whether to demand the resistance to gravelling is demonstrated.

\subsubsection{Gravelling impact resistance}

\subsubsection{Test piece mounting}

The test piece shall be placed on a rigid steel mounting with a cutout of size $460 \mathrm{~mm} \times$ $460 \mathrm{~mm}$, and an outer frame holding the piece on all four sides (see Fig. 1A). A strip of hard (50 D.I.D.C. or 46 Shore) neoprene $2 \mathrm{~mm}$ thick and $20 \mathrm{~mm}$ wide shall be placed between the mounting, the outer frame and test piece. The glass shall be thus secured to a depth of $20 \mathrm{~mm}$. The outer frame shall be fitted and held in position so as to hold the test piece securely without deforming it, with at least two fastening points on each side.

\subsubsection{Projectile}

The projectile (see Fig. 1B) shall be made of aluminium alloy according to ISO 6362-2:1990, grade 2017A, with a nominal weight of $20 \mathrm{~g}$ and dimensions as shown in Fig. 1B.
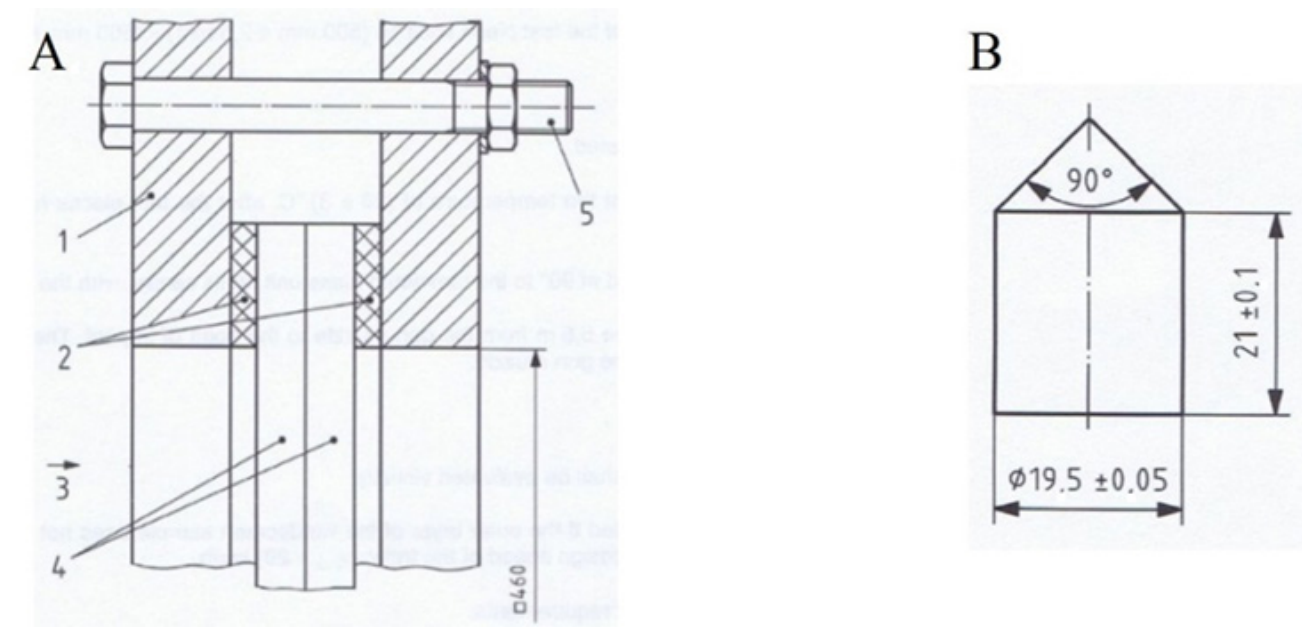

Fig. 1. A-Test piece holder: 1 -outer frame, 2 - neoprene strips, 3 - direction of impact, 4 -test piece, 5-mounting; $B$ - Projectile

\subsubsection{Test piece}

The nominal dimensions of the test piece shall be $(500 \mathrm{~mm} \pm 2.5 \mathrm{~mm}) \times(500 \mathrm{~mm} \pm 2.5 \mathrm{~mm})$.

\subsubsection{Test method}

Three samples shall be tested. Projectiles shall be fired at the temperature of $(20 \pm 3)^{\circ} \mathrm{C}$, after the test pieces have been kept at $20^{\circ} \mathrm{C}$ for $24 \mathrm{~h}$. The projectile shall be fired at $90^{\circ}$ to the laminated glass unit, at its centre, with the point forwards. The firing distance shall be $5.5 \mathrm{~m}$ from the gun muzzle to the point of impact. The projectile's speed shall be measured at $2.5 \mathrm{~m}$ from the gun 
muzzle. The effect of the impacts shall be evaluated visually. Compliance is demonstrated if the outer layer of the windscreen sample does not break at a projectile speed $20 \mathrm{~km} / \mathrm{h}$ greater than the design speed of the train: $(\operatorname{Vmax}+20) \mathrm{km} / \mathrm{h}$.

\section{Initial tests}

In order to prepare for the gravelling test in accordance with the standard requirements [6], 12 test shots were made. Three of them were recorded with a high-speed camera (see Fig. 2). The impact tests were carried out using a prepared sub-calibre projectile and the pneumatic cannon [1] normally used to make shots in accordance with the requirements of UIC-651 [8]. The projectiles with visible dents after hitting the glass are shown in Fig. 3. The angle between the axis of the projectile and the vector perpendicular to the sample plane was determined visually. Projectiles were placed on grid paper, and the impact angle was determined using an adjustable protractor.
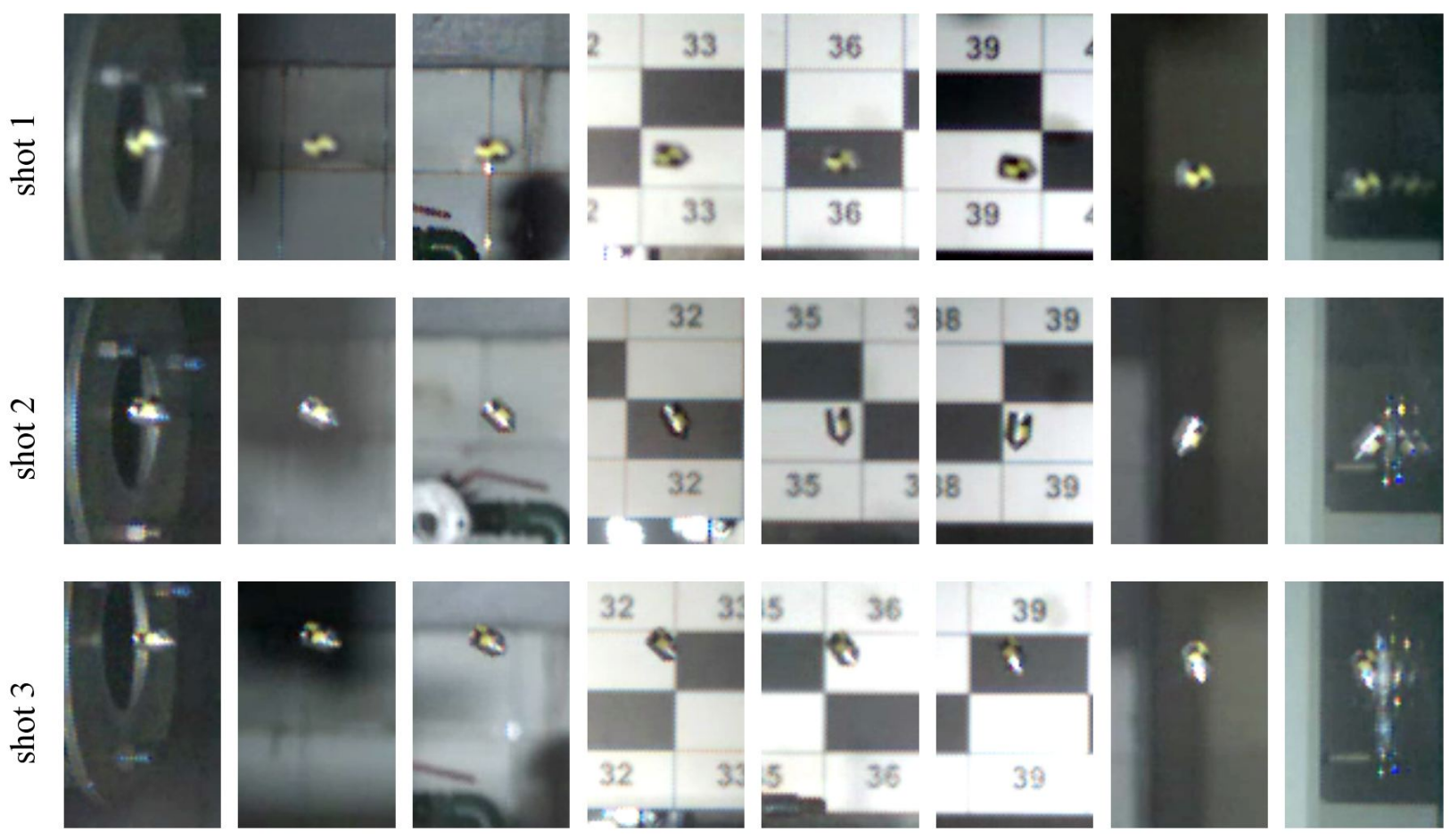

Fig. 1. Trajectory of projectile, performed in accordance with the standard guidelines [6], during 3 impact tests recorded with a high-speed camera
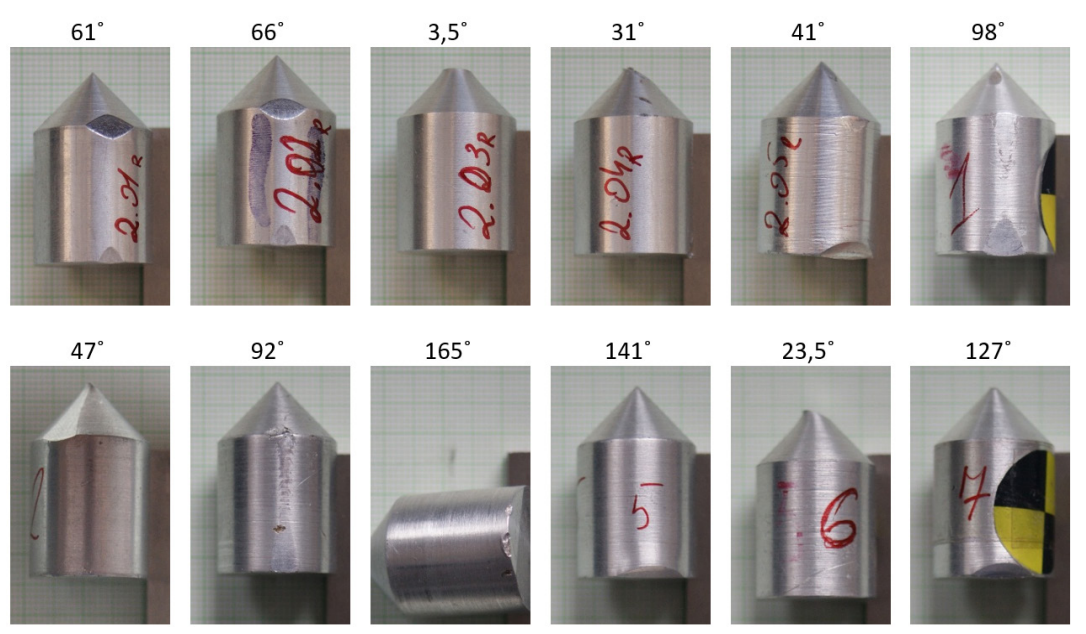

Fig. 2. Projectiles made in accordance with the requirements of standard [6] after the impact test with visible dents 


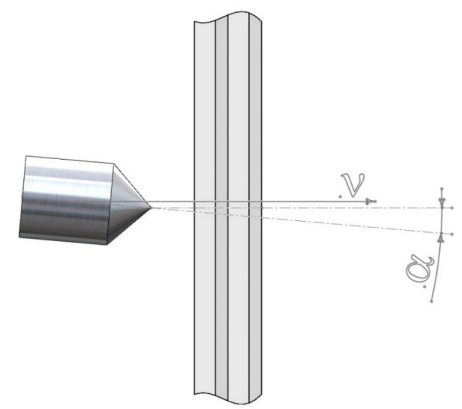

Fig. 3. Sketch of the projectile impact on the glass sample with deviation by angle $\alpha$ from perpendicularity; $v$ - projectile velocity, $\alpha$-impact angle

\section{Projectile shape}

The reason why the projectile rotates after leaving the cannon barrel is the position of the centre of mass $(\mathrm{CM})$ located behind the centre of aerodynamic pressure $(\mathrm{CP})$ of the projectile. The design distance between $\mathrm{CM}$ and $\mathrm{CP}$ is $1 \mathrm{~mm}$. In this arrangement, even at the slightest angular deviation of the projectile axis from the direction of aerodynamic pressure, resultant torque increases the projectile deviation (Fig. 5A). Such a projectile is by definition unstable [3]. The change of position of these characteristic points in relation to each other and their proper distance cause that the resultant torque stabilises the projectile in the direction of the inertia force driving it (Fig. 5B).
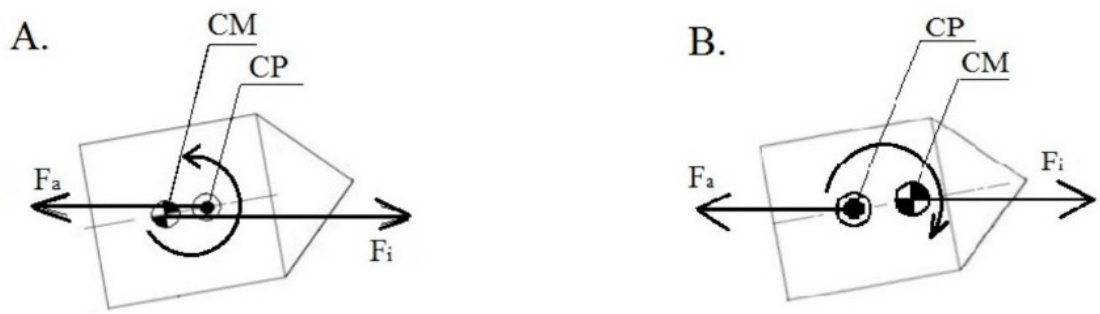

Fig. 4. Projectile shape according to PN-EN 15152:2007-force distribution: A - unstable, B - stable; where: $C M$ - centre of mass, $C P$-centre of aerodynamic pressure, $F_{a}$-aerodynamic force, $F_{i}$-inertia force

A similar problem occurs in military applications. Projectiles tend to take a backward position. To stabilise projectiles a gyroscopic phenomenon is used. A special thread on the inner wall of the barrel causes the projectile high rotation speed (even $200000 \mathrm{rpm}$ [3]). In smoothbore weapons projectiles, , the centre of mass is moved forward by hollowing out the projectile at the rear in order to stabilise them, or the centre of aerodynamic pressure is moved backwards by adding a light stabilising element ("tail") to the bottom [3].

A similar problem with stability occurs in simple rockets, where flight stabilisation is carried out exclusively by fins [1]. For such rockets, wind tunnel tests are carried out in order to determine the position of the aerodynamic centre depending on the angle of attack [5].

\section{Modified projectile tests}

In order to stabilise the trajectory of the projectile, 4 types of projectiles were made using military experience. According to the authors, the modified projectiles retained all the important parameters in order to preserve the idea of a gravelling test described in the standard [6]. Therefore, similarly to the smoothbore weapon bullets [3], the projectiles shown below were made (Fig. 6). It was assumed that the result of the gravelling test is influenced only by the projectile parameters given below and the projectile velocity. The modified projectiles retained the following properties: 
- Material - Al 2107A aluminium alloy,

- Apex shape $\left(90^{\circ}\right.$ cone $)$ and diameter $(\phi 19.5 \pm 0.05 \mathrm{~mm})$,

- Weight $20 \mathrm{~g}$.
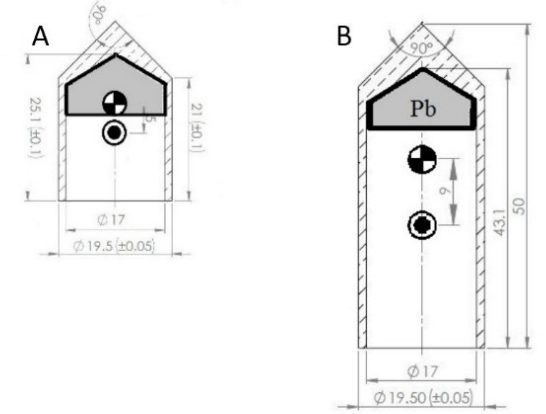

- Centre of Mass (CM)

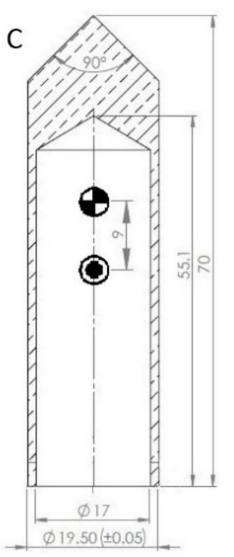

- Centre of Pressure (CP) (indicative position)

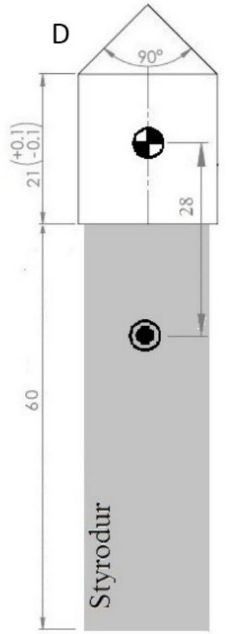

Fig. 5. Modified projectiles used during the tests: A - Projectile with external dimensions according to the standard [1], hollowed and loaded with lead up to $20 \mathrm{~g} ; \mathrm{B}$-Expanded projectile up to $50 \mathrm{~mm}$, hollowed and loaded with lead up to $20 \mathrm{~g}$; $\mathrm{C}$-Expanded projectile up to $70 \mathrm{~mm}$, hollowed to the depth corresponding to a mass of $20 \mathrm{~g} ; \mathrm{D}$-Projectile made in accordance with the guidelines of the standard, with a styrodur cylinder affixed $20 \mathrm{~g}+0.4 \mathrm{~g}$

The impact tests were carried out at a speed of about $140 \mathrm{~km} / \mathrm{h}$. The centre of the projectile mass was determined using a 3D model made in SolidWorks. The approximate CP position of the projectile was determined by a simplified 'flat projection weighting method', which is a determination of the centre of mass for the flat side projection of the projectile.

\section{Results of the projectile modified tests}

A number of tests were made with the projectiles presented above (Fig. 6). The tests were not recorded with a high-speed camera. Only the denting left on the projectile after hitting the glass was assessed visually (see Initial tests).
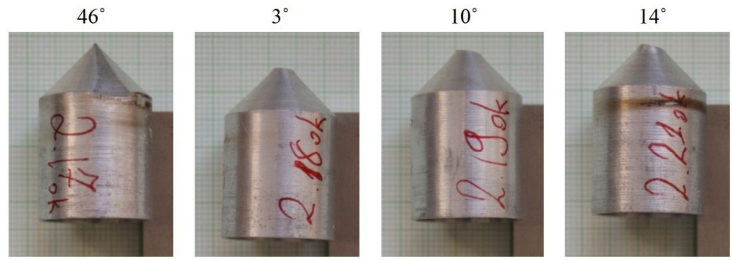

Fig. 6. Short hollow projectiles after the impact test
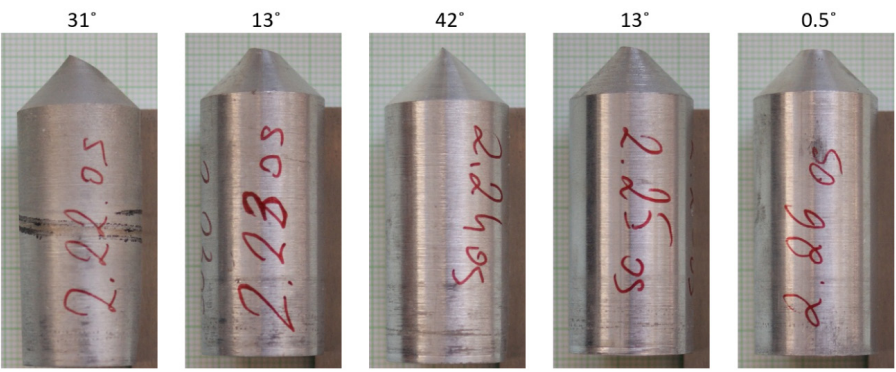

Fig. 7. Medium hollow projectiles after the impact test 

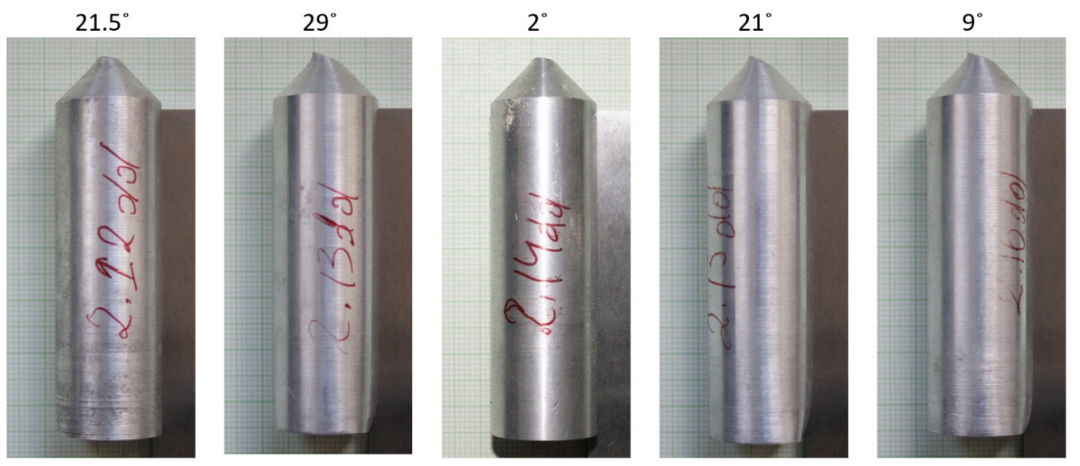

Fig. 8. Long hollow projectiles after the impact test
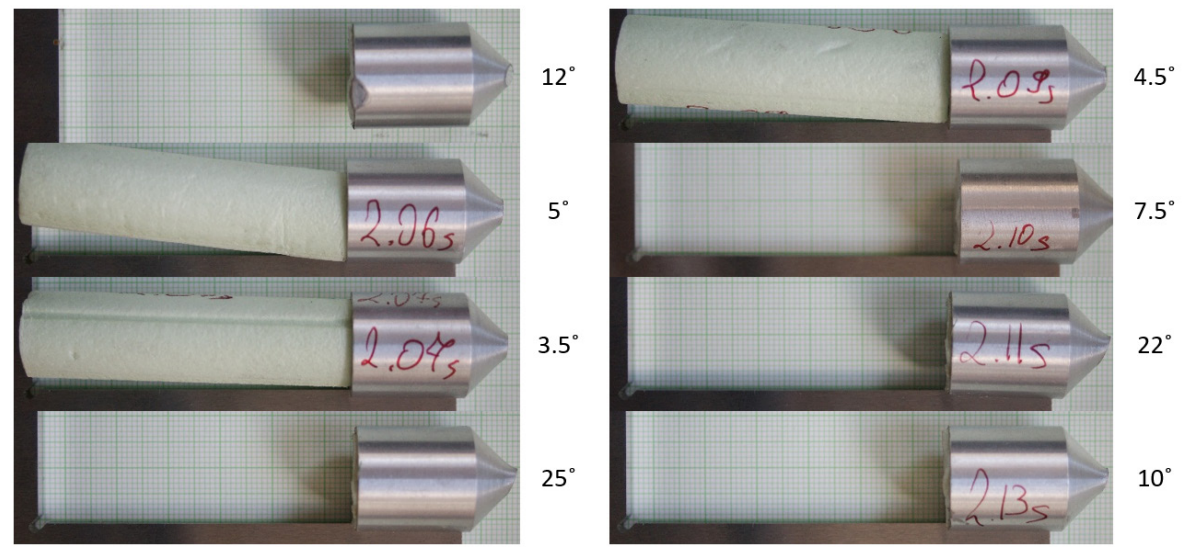

Fig. 9. Projectiles with guidance after the impact test

\subsection{Short hollow projectile with lead}

The first modification of the projectile consisted in drilling in the rear part of the projectile, made according to the requirements of the standard [6], and then partially filling the hole with the amount of lead measured in order to obtain the projectile mass of $20 \mathrm{~g}$ (Fig. 6A). This procedure allowed moving the $\mathrm{CM}$ about $6 \mathrm{~mm}$ forward. In this configuration, analytical $\mathrm{CM}$ is located $5 \mathrm{~mm}$ in front of CP. Four impact tests were carried out in the configuration for which an average deviation of the expected impact angle $\alpha$ of $18^{\circ}$ was obtained, with the variance of the results being $16.5^{\circ}$ (see Fig. 7).

\subsection{Medium hollow projectile with lead}

The second modification of the projectile was to extend the projectile from the standard [6] to $50 \mathrm{~mm}$. The projectile was also drilled in and loaded with lead in the front part so that its total mass was $20 \mathrm{~g}$ (Fig. 6B). In this configuration CM is $9 \mathrm{~mm}$ in front of CP. Five impact tests were carried out in this configuration, for which an average deviation of the expected impact angle $\alpha$ of $20^{\circ}$ was obtained, with the variance of the results being $15^{\circ}$ (see Fig. 8).

\subsection{Long hollow projectile}

The third modification of the projectile consisted in extending the projectile from the standard [6] to $70 \mathrm{~mm}$. The weight of $20 \mathrm{~g}$ was achieved by drilling $55 \mathrm{~mm}$ deep in the rear (Fig. 6C). In this configuration $\mathrm{CM}$ is $9 \mathrm{~mm}$ in front of CP. Five impact tests were made in this configuration, for which an average deviation of the expected impact angle $\alpha$ of $16.5^{\circ}$ was obtained, with the variance of the results being $10^{\circ}$ (see Fig. 9). 


\subsection{Projectiles with a stabiliser}

The fourth projectile modification consisted in sticking a cylinder made of Styrodur 3035 CS with a diameter of $16.5 \mathrm{~mm}$ and a length of $60 \mathrm{~mm}$, weighing approximately $0.4 \mathrm{~g}$, to the projectile from the standard [6] (Fig. 6D). The stabiliser increased the projectile mass by about $0.4 \mathrm{~g}$, but that did not have a significant impact on the purposes of the projectiles tests. In this configuration CM is located $28 \mathrm{~mm}$ in front of CP. Eight impact tests were carried out in this configuration, for which an average deviation of the expected impact angle $\alpha$ of $11^{\circ}$ was obtained, with the variance of the results being $8^{\circ}$ (see Fig. 10). In some projectiles, the tail part of the projectile detached from it after rebounding from the glass.

\section{Inaccuracies in the standard}

According to the authors, the content of the standard concerning the gravelling test should be clarified in the following matters:

- Specification of the permissible deviations for the impact angle of the projectile on the glass. The method of measuring the actual angle of the projectile impact on the glass should also be specified.

- Specification of permissible projectile mass deviations.

- Specification of the strip. The standard specifies the hardness of the strip and its material. Unfortunately, the name of the material mentioned in the standard i.e., Neoprene is not precise enough. The hardness scales of the required Neoprene given in the standard are not precise, either, i.e. it is not specified exactly what type of Shore hardness scale the authors of the standard had in mind. Different scales (Shore A; Shore D; Shore AO; Shore AM, etc.) are used to measure the hardness of non-metals due to their long span of hardness [4].

\section{Conclusions}

The results of the impact tests presented above confirm that the transfer of the CM in front of CP significantly improves the stability of the projectile flight (see Table 1). Each of 22 modified projectiles hit the test piece with the tip, while out of the 12 original projectiles only 4 hit the test piece with the tip. The effect of the CM-CP distance is also clearly visible. For the longest modification with a stabiliser (CM-CP distance: $28 \mathrm{~mm}$ ), the average deviation from the vertical of the angle of impact was $11^{\circ}$, the maximum deviation was $25^{\circ}$ (see Fig. 10), and for the shortest (CM-CP distance: $5 \mathrm{~mm}) 18^{\circ}$ and $46^{\circ}$ respectively. Extending the length of the stabiliser is likely to give even better results.

Only 34 impact tests were made, but such a small amount of data confirms the assumed dependence of the effect of the CM-CP position on the stability of the projectile flight at relatively low flight speed.

Tab. 1. Test results

\begin{tabular}{|c|c|c|c|}
\hline Type & Distance CM to $\mathbf{C P}[\mathbf{m m}]$ & Average $\boldsymbol{\alpha}\left[{ }^{\circ}\right]$ & Variance $\left[{ }^{\circ}\right]$ \\
\hline Stabilised & 28 & 11 & 8 \\
\hline Long & 9 & 16.5 & 10 \\
\hline Medium & 9 & 20 & 15 \\
\hline Short & 5 & 18 & 16.5 \\
\hline Standard & -1 & 48 & 75 \\
\hline
\end{tabular}




\section{Recommendations}

In the authors' opinion, a change in the guidelines concerning the measurement point of velocity and the distance of the tested sample from the end of the cannon barrel should also be considered. At projectile velocities between $120 \mathrm{~km} / \mathrm{h}$ and $180 \mathrm{~km} / \mathrm{h}$, the trajectory of the projectile already clearly falls down, which is also connected with slight change in the intersection angle of the projectile trajectory with the tested glass pane. The authors propose to reduce the distance from the end of the barrel to the glass by $2.5 \mathrm{~m}$.

The authors propose the projectile geometry with a stabiliser with a length of $100 \mathrm{~mm}$ (Fig. 11). It is also suggested to collect the centring of the stabiliser in relation to the projectile's axis, which at the same time will allow achieving the projectile weight determined in the standard [6] requirements $(20 \mathrm{~g})$.

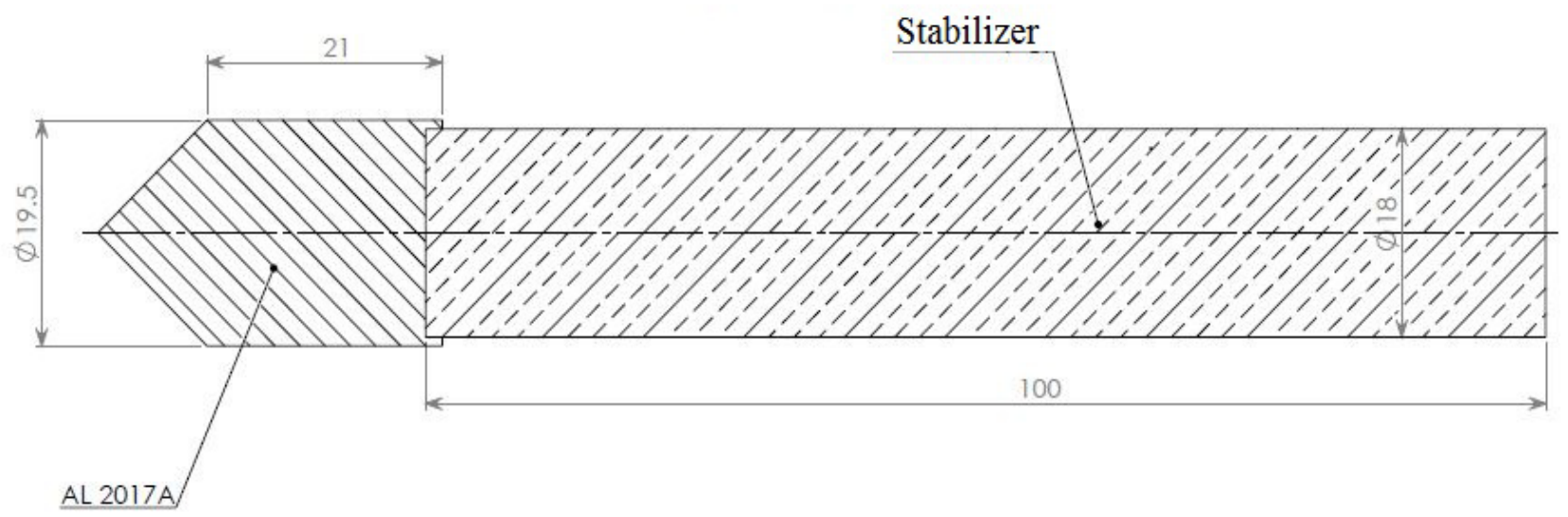

Fig. 10. Sketch of the proposed modification of the projectile for further testing

During a gravelling test, the projectile undergoes plastic deformation due to the impact on the glass. This deformation is so pronounced that it can be used to assess the correctness of the impact test. It is worth supplementing the standard with a method to assess the deformation of the projectile after the test.

\section{Acknowledgements}

The authors express his gratitude to colleagues from the Institute of Aviation, thanks to whose courtesy this article was written, in particular: Tomasz Dörre, Mirosław Kozera, Grażyna Kubiś, Borys Lukasik. Special thanks are owned to Aleksandra Jakubiak for language polishing services. Thanks are also due to the management of Rail-Bohamet who carried out samples of windscreens for the purpose of testing.

\section{References}

[1] Boguszewicz, P., Pneumatic gun for testing impact perforation resistance of railway windshield, Tech. Transp. Szyn., No. 12, pp. 172-176, 2015.

[2] Cieśliński, D., Precise determination of static margins for unguided sounding rockets, In: 66th International Astronautical Congress, paper no. IAC-15.E2.1.4.x30641, Jerusalem, Israel 2015.

[3] Ejsmont, J., Balistyka dla snajperów: praktyczny poradnik, Wydawnictwa Naukowo-Techniczne, pp. 91-95, Warsaw 2011.

[4] ISO 48-4:2018 2018 Rubber, vulcanized or thermoplastic - Determination of hardness Part 4: Indentation hardness by durometer method (Shore hardness). 
[5] Marciniak, B, Okninski, A, Bartkowiak, B, et al., Development of the ILR-33 "Amber" sounding rocket for microgravity experimentation, Aerosp Sci Technol, Vol. 73, pp. 19-31, 2018.

[6] PN-EN 15152:2007, Railway applications - Front windscreens for train cabs.

[7] Szafran, K., Traction vehicle operator safety - laboratory dynamic tests, Logistyka, No. 6, 10192-10197, 2014.

[8] UIC 651, Layout of driver's cabs in locomotives, railcars, multiple-unit trains and driving trailers.

Manuscript received 14 December 2018; approved for printing 18 March 2019 